\title{
ELETROESTIMULAÇÃO E PACIENTES COM DOENCA PULMONAR OBSTRUTIVA CRÔNICA: um relato de casos
}

\author{
Carla Sousa BORGES ${ }^{1}$
}

\author{
Lilian Cristina Gomes do NASCIMENTO²
}

Maria Georgina Marques TONELLO ${ }^{3}$

Juliana Ribeiro Gouveia REIS ${ }^{4}$

\begin{abstract}
${ }^{1}$ Aluna do curso de Fisioterapia - Centro Universitário de Patos de Minas, Patos de Minas, MG, Brasil.carla.sb@hotmail.com

${ }^{2}$ Doutoranda em Promoção da Saúde - Universidade de Franca, Franca, SP, Brasil.liliangomes@ hotmail.com.br

${ }^{3}$ Docente do Programa de Pós Graduação Promoção da Saúde - Universidade de Franca, Franca, SP, Brasil.gina@ginatonello.com

${ }^{4}$ Docente do curso de Fisioterapia do Centro Universitário de Patos de Minas, Patos de Minas, MG, Brasil.julianargr@hotmail.com
\end{abstract}

Recebido em: 04/10/2015 - Aprovado em: 17/07/2016 - Disponibilizado em: 18/12/2016

\section{RESUMO}

A doença pulmonar obstrutiva crônica (DPOC) classifica-se como importante causa de morbimortalidade no mundo, em relação a eletroestimulação, este é um recurso que visa melhorar a força da musculatura. O objetivo desse trabalhofoiverificar os efeitos de um protocolo de eletroestimulação na recuperação da musculatura respiratória em pacientes com DPOC. Participaram deste estudo dois pacientes que foram submetidos à fisioterapia convencional associada à eletroestimulação respiratória, duas vezes por semana durante 08 semanas. Foram avaliadas as pressões respiratórias máximas utilizando o manovacuômetro e a qualidade de vida através do questionário do hospital Saint George,antes e após o tratamento. Observou-se que os dois pacientes apresentaram aumento na PImáx (caso 1:de -35 $\mathrm{cmH}_{2} \mathrm{O}$ para $-50 \mathrm{cmH}_{2} \mathrm{O}$, representando uma evolução de $43 \%$; caso 2: de $-80 \mathrm{cmH}_{2} \mathrm{Opara}-120 \mathrm{cmH}_{2} \mathrm{O}$ equivalente a um ganho de 50\%) e na PEmáx (caso 1: de $40 \mathrm{cmH}_{2} \mathrm{O}$ para $70 \mathrm{cmH}_{2} \mathrm{O}$, aumentando 75\%; caso 2:de $90 \mathrm{cmH}_{2} \mathrm{O}$ para $100 \mathrm{cmH}_{2} \mathrm{O}$, evoluindo11\%) e na qualidade de vida, principalmente nos quesitos atividades (caso 1: 81\%; caso 2: 67\%) e impacto (caso 1: 50\%; caso 2: 100\%). Consideramos que a aplicação do protocolo de eletroestimulação associado à fisioterapia convencional, pode melhorar aforça da musculatura respiratória e qualidade de vida de pacientes.
\end{abstract}

Palavras-chave: Doença pulmonar. Qualidade de vida. Estimulação elétrica.

\begin{abstract}
Chronic obstructive pulmonary disease (COPD) is considered as an important cause of morbidity and mortality in the world.Electrical stimulation is a physiotherapy resource aimed improving muscle strength.The aim of this study was to assess the effects of an electrical stimulation protocol recovery of the respiratory muscles of patients with COPD.The study included two patients who underwent conventional physiotherapy associated with respiratory electrical stimulation twice a week for 08 weeks. Was measuredmaximal respiratory pressuresusing themanometerandthe quality of lifethrough thehospitalquestionnaireSaintGeorge, before andafter treatment.It was observed that two patients had an increase in MIP (case 1: -35 to $-50 \mathrm{cmH} 2 \mathrm{O} \mathrm{cmH} 2 \mathrm{O}$, representing an increase of $43 \%$; case 2: -120 to $-80 \mathrm{cmH} 2 \mathrm{O}$ $\mathrm{cmH} 2 \mathrm{O}$ equivalent to a 50\% gain) and $\mathrm{MEP}$ (case 1: $40 \mathrm{cmH} 2 \mathrm{O}$ to $70 \mathrm{cmH} 2 \mathrm{O}$, increasing $75 \%$; case 2: $90 \mathrm{cmH} 2 \mathrm{O}$ to $100 \mathrm{cmH} 2 \mathrm{O}$, progressing 11\%) and quality of life in the categories activities (case 1: 81\%; case $2: 67 \%$ ) and impact (case 1: 50\%; case 2: 100\%).We consider that application of electrical stimulation protocol associated with conventional physical therapy, can improve respiratory muscle strength and quality of life of these patients.
\end{abstract}

Keywords: Pulmonary disease. Quality of life. Electricalstimulation. 


\section{INTRODUÇÃO}

A doença pulmonar obstrutiva crônica (DPOC) classifica-se atualmente como importante causa de morbimortalidade no mundo, sendo esta caracterizada por uma obstrução não reversível ao fluxo aéreo e perda progressiva da função pulmonar (FERNANDES, 2009).

A DPOC é uma doença progressiva e não reversível com medicação broncodilatadora, uma manifestação comum no paciente DPOC é a limitação progressiva ao exercício físico devido à disfunção da musculatura esquelética caracterizada pela redução da massa magra e hipotrofia das fibras tipo I e IIA (ARAUJO; SANTOS, 2012), sendo a dispneia é um dos principais sintomas da DPOC e frequentemente interfere em vários aspectos da vida do paciente, como nas atividades profissionais, familiares $\mathrm{e}$ sociais (SBPT, 2012).

O tratamento da DPOC abrange uma série de recursos como, mudanças comportamentais, fisioterapia respiratória, tratamento farmacológico e exercício físico. A eletroestimulação neuromuscular (EENM) é utilizada na prevenção da atrofia causada pelo desuso. A EENM age por meio de impulsos elétricos despolarizando a membrana, gerando um potencial de ação que se propaga no nervo até atingir o músculo, com a mesma intensidade inicial fazendo com que ele
contraia.Existem relatos que comprovam que através da EENM pode-se ativar de $30 \%$ a $40 \%$ mais unidades motoras durante a estimulação com correntes de média frequência se comparado ao movimento ativo (ARAUJO; SANTOS, 2012).

A realização desse trabalho justificou-se, pois ainda hoje existem muitas interrogações acerca do uso da eletroestimulação no tratamento de disfunções diafragmáticas, sendo que essa técnica é pouco difundida, pouco utilizada e estudada no meio acadêmico e profissional (SILVA; VIEIRA, 2009). Assim, este trabalho teve como objetivo avaliar os efeitos corrente elétrica funcional (FES) na força da musculatura respiratória verificada através da manovacuometria e identificar alterações na QV.

\section{METODOS}

O presente estudo foi submetido ao Comitê de Ética do Centro Universitário de Patos de Minas, aprovado sob protocolo $\mathrm{n}^{\circ}$ 683.895. Selecionou-se indivíduos com diagnóstico clínico de DPOC e fraqueza da musculatura respiratória, com idade superior a 60 anos. Sendo excluídos aqueles que apresentassem qualquer tipo de instabilidade 
hemodinâmica ou queda de saturação durante a execução do protocolo.

Mediante esses critérios, enquadraram-se na pesquisa 02 pacientes, que consentiram em participar,assinando o Termo de Consentimento Livre e Esclarecido.

\section{Avaliação da Musculatura Respiratória}

Inicialmente foram verificadas a pressão arterial (mmHg), saturação periférica de oxigênio (\%) e frequência cardíaca (bpm).

A determinação de PImáx e PEmáx foi mensurada para diagnóstico inicial de fraqueza dos músculos respiratórios e posterior reavaliação ao final do protocolo para comparação dos resultados, mediante a utilização da manovacuometria.Durante a realização das medidas, os pacientes estavam sentados e utilizando um clipe nasal, utilizouse um manovacuômetro analógico marca Wika, e estes foram orientados a realizarem uma inspiração e uma expiração forçada máxima. Esse processo foi realizado em triplicata, das quais selecionamos a de maior valor.

\section{Qualidade de vida}

O nível da QV dos pacientes avaliados foi obtido por meio da aplicação do Questionário do Hospital Saint George de qualidade de vida (SGRQ) (SOUSA; JARDIM; JONES, 2000), que foi respondido pelos próprios participantes do estudo, no inicio e ao final da execução do protocolo.

\section{Protocolo}

Os pacientes receberam cuidados de fisioterapia respiratória e motora convencional duas vezes por semana, durante 8 semanas,que incluiu: manobras de higiene brônquica (tapotagem e vibração manual),exercícios respiratórios (frênio-labial e EPAP selo d'aguae alongamentos (peitoral e bíceps braquial). Após a realização da fisioterapia convencional, utilizou-se o aparelho Fesvil 995 da marca Quark para submeter os pacientes à eletroestimulação de baixa voltagem.

Os pacientes foram posicionados em decúbito dorsal, com tronco elevado a $45^{\circ}$ de flexão, para esta angulação foi utilizado o trapézio da marca J. B.. Foram utilizados quatro eletrodos de silicone, adaptados na pele com gel hidrossolúvel, e fixados com fita micropore $(3 \mathrm{M})$. Foram posicionados dois eletrodos em cada lado do tórax, entre a $2^{\mathrm{a}}$ e a $6^{\text {a }}$ costela, estimulando os músculos intercostais internos e externos, responsáveis respectivamente pela expiração forçada e inspiração, e o diafragma, principal músculo 
responsável pela respiração

(CANCELLIERO; COSTA; SILVA, 2006).

Os parâmetros foram ajustados da seguinte maneira: frequência de $30 \mathrm{~Hz}$; largura de pulso (T) de 40ms; sustentação de $1 \mathrm{~s}$ e repouso de $1 \mathrm{~s}$, por 30 minutos $\mathrm{A}$ intensidade da corrente foi à mínima para obter a contração do músculo diafragma, causando sensação confortável à voluntária.

\section{Análise dos dados}

Os dados foram registrados em uma planilha e posteriormente analisados mediante comparação em valores absolutos e percentuais, bem como a partir da confecção de gráficos, utilizando o programa Microsoft Office Excel 2010.

\section{APRESENTAÇÃO DOS CASOS}

\section{Caso 1}

Participante 1, 70 anos, sexo masculino, aposentado, ex-tabagista, residente em Patos de Minas. Apresenta diagnóstico clínico de asma e DPOC, e fisioterapêutico de fraqueza da musculatura respiratória, fadiga e dispnéia, com via de entrada de ar oral. Há 10 anos apresentou quadro de forte dispneia e ao ser levado ao hospital diagnosticou-se asma e DPOC. A partir daí seguiu fazendo acompanhamento constante.Há 15 dias apresentou muita falta de ar, tosse e cansaço, não conseguindo realizar as atividades rotineiras, nem dormir, sendo levado ao hospital, permaneceu sob medicação e oxigenoterapia, por 08 dias, período no qual se detectou um agravo na patologia de base.

\section{Caso 2}

Participante 2, 65 anos, sexo masculino, motorista, tabagista, residente em Patos de Minas. Diagnóstico clínico de DPOC e fisioterapêutico de fraqueza da musculatura respiratória,com via de entrada de ar mista. Há 05 anos em consulta de rotina ao clínico geral relatou ser fumante, com isso foi solicitado um raio $\mathrm{X}$ onde se visualizou uma "mancha" no pulmão, conforme relato do próprio paciente, ele foi encaminhado a um pneumologista, o qual solicitou uma tomografia e espirometria, detectando a DPOC.

\section{RESULTADOS}

Os valores da avaliação da força muscular respiratória pela medida de PImáx e PEmáx antes da aplicação do protocolo, estão apresentadas no Gráfico 1, pode-se notar a presença de déficit na força muscular dos pacientes. 
Gráfico 1 - Avaliação da força muscular respiratória pela medida de PImáx e PEmáx antes da aplicação do protocolo

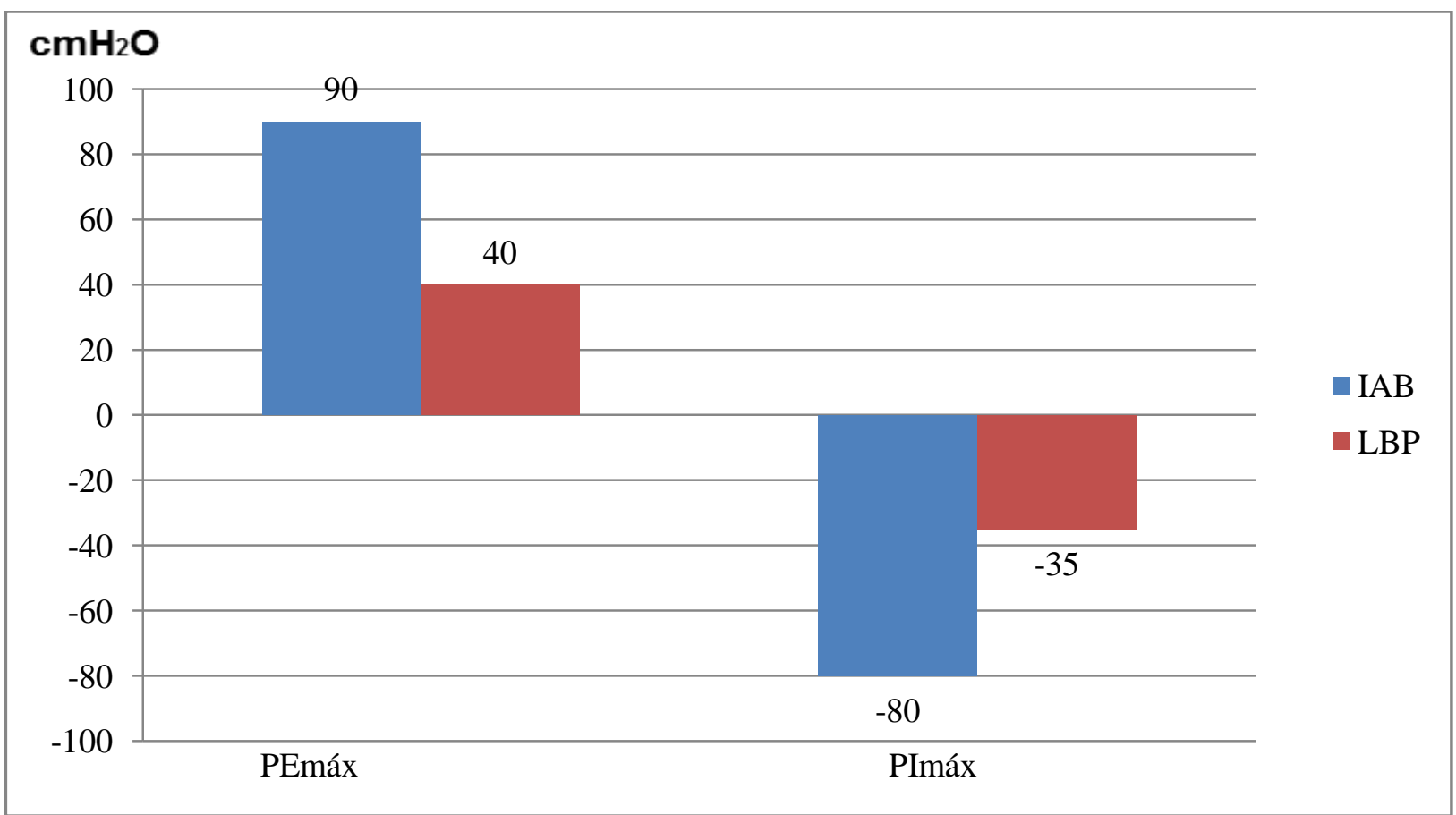

Fonte: elaborada pelos autores

Considerando os achados desse daPImáx e PEmáx, em ambos os casos estudo, notou-se um aumento nos valores (Gráficos 2 e 3).

Gráfico 2 - Comparação das medidas antes e após aplicação do protocolo de eletroestimulação no caso 1.

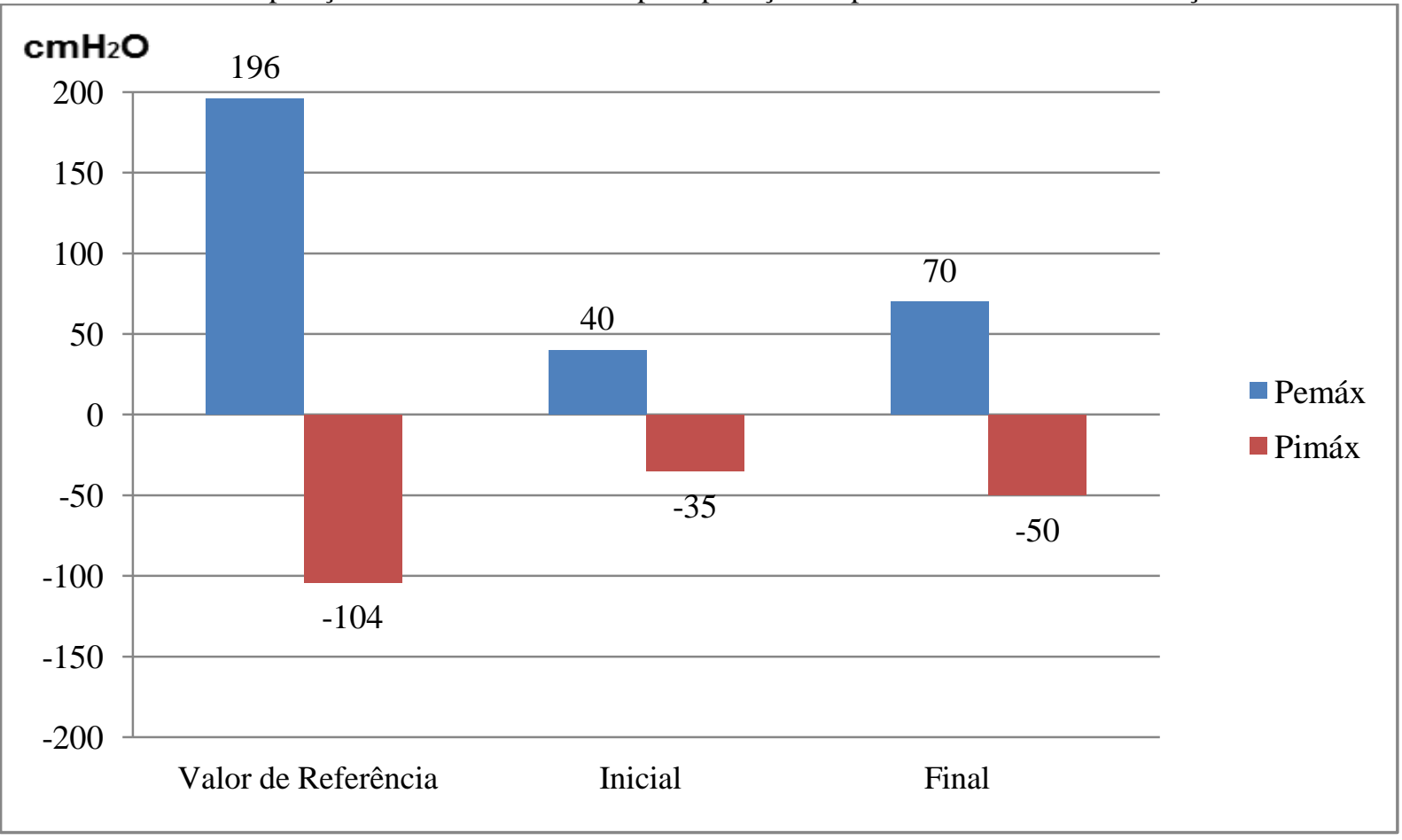

Fonte: elaborada pelos autores 
Gráfico 3 - Comparação das medidas antes e após aplicação do protocolo de eletroestimulação no caso 2.

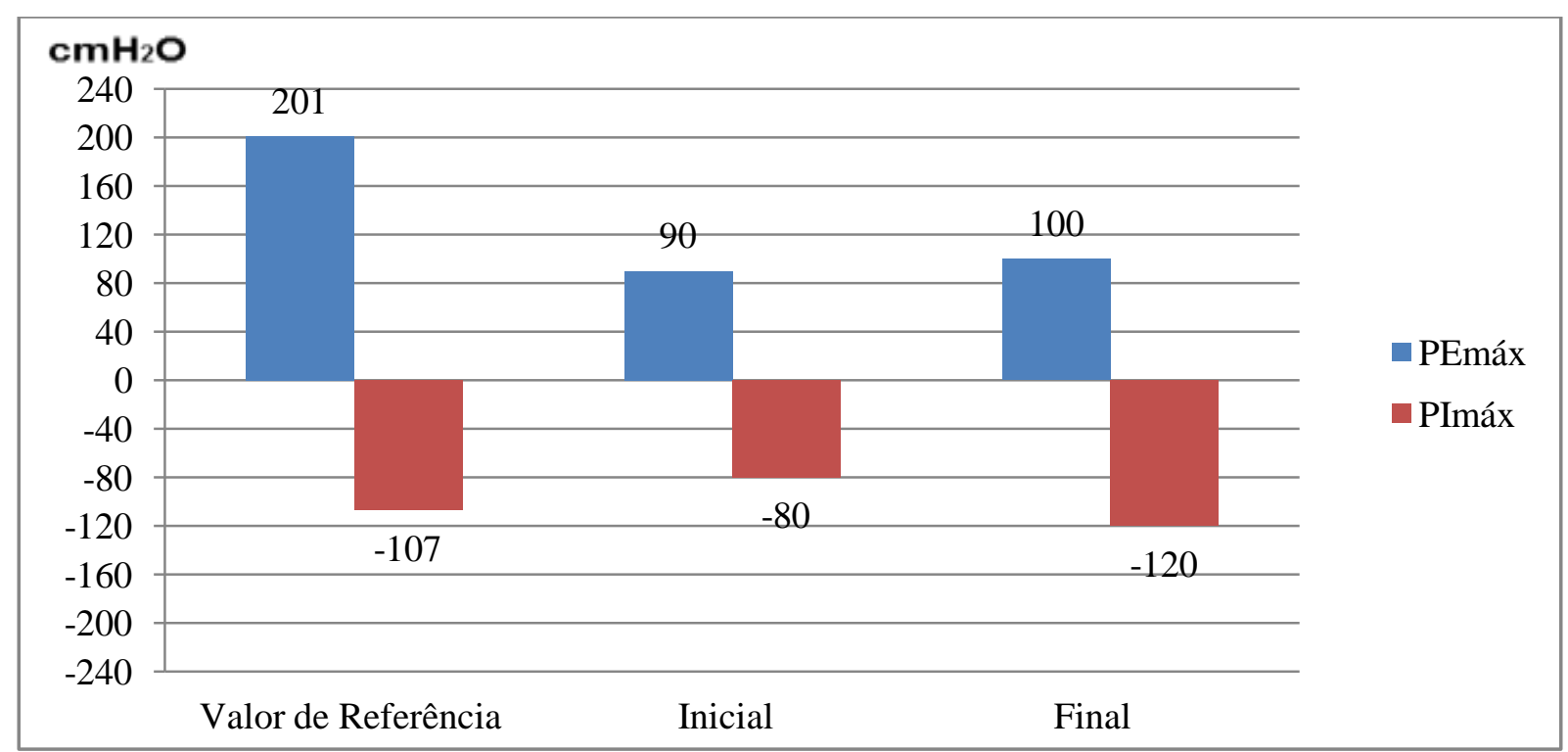

Fonte: elaborada pelos autores

Os gráficos 4 e 5 trazem cada domínio do questionários nos casos 1 e respectivamente a somatória dos pontos de 2.

Gráfico 4 - Comparação dos somatórios inicial e final dos domínios do questionário de qualidade de vida do caso 1

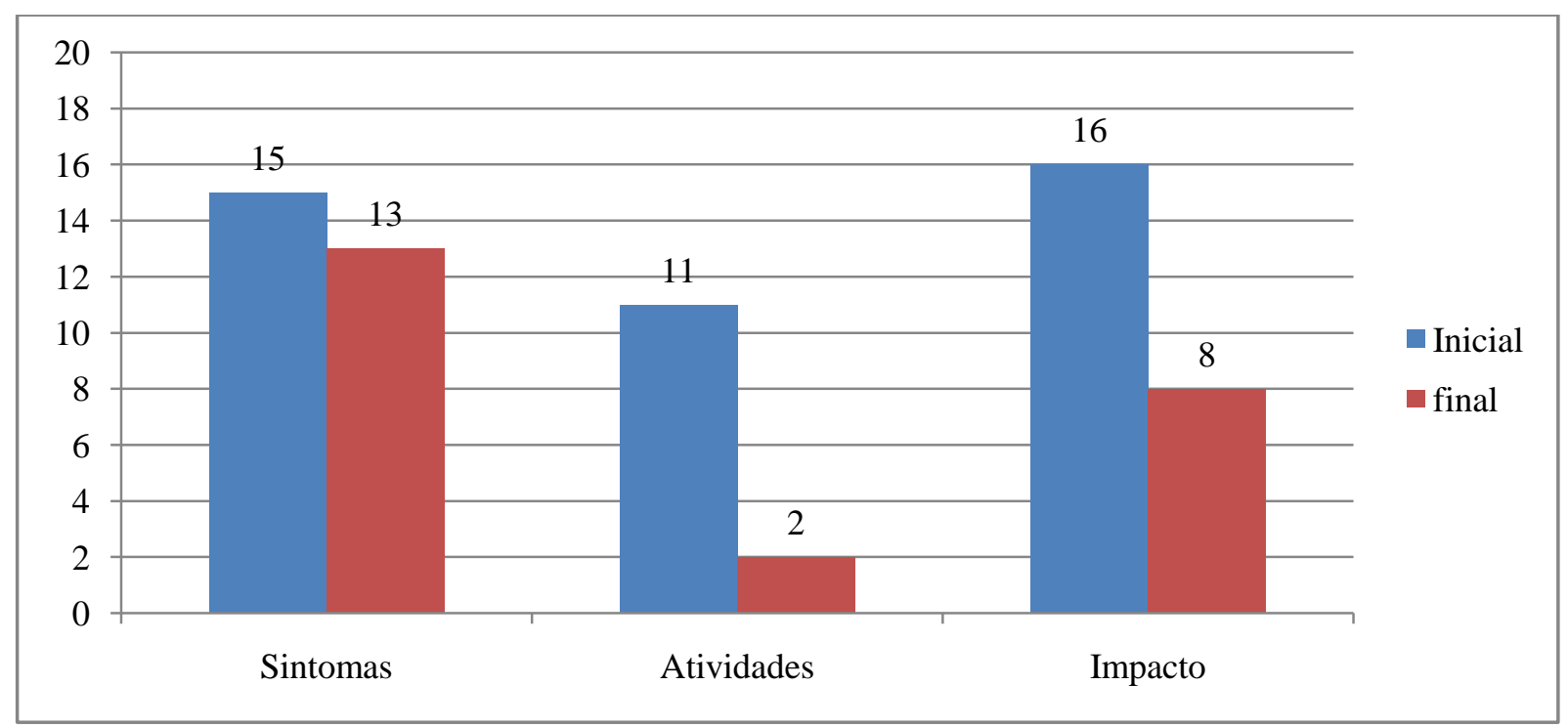

Fonte: elaborada pelos autores 
Gráfico 5 - Comparação dos somatórios iniciais e finais dos domínios do questionário de qualidade de vida do caso 2

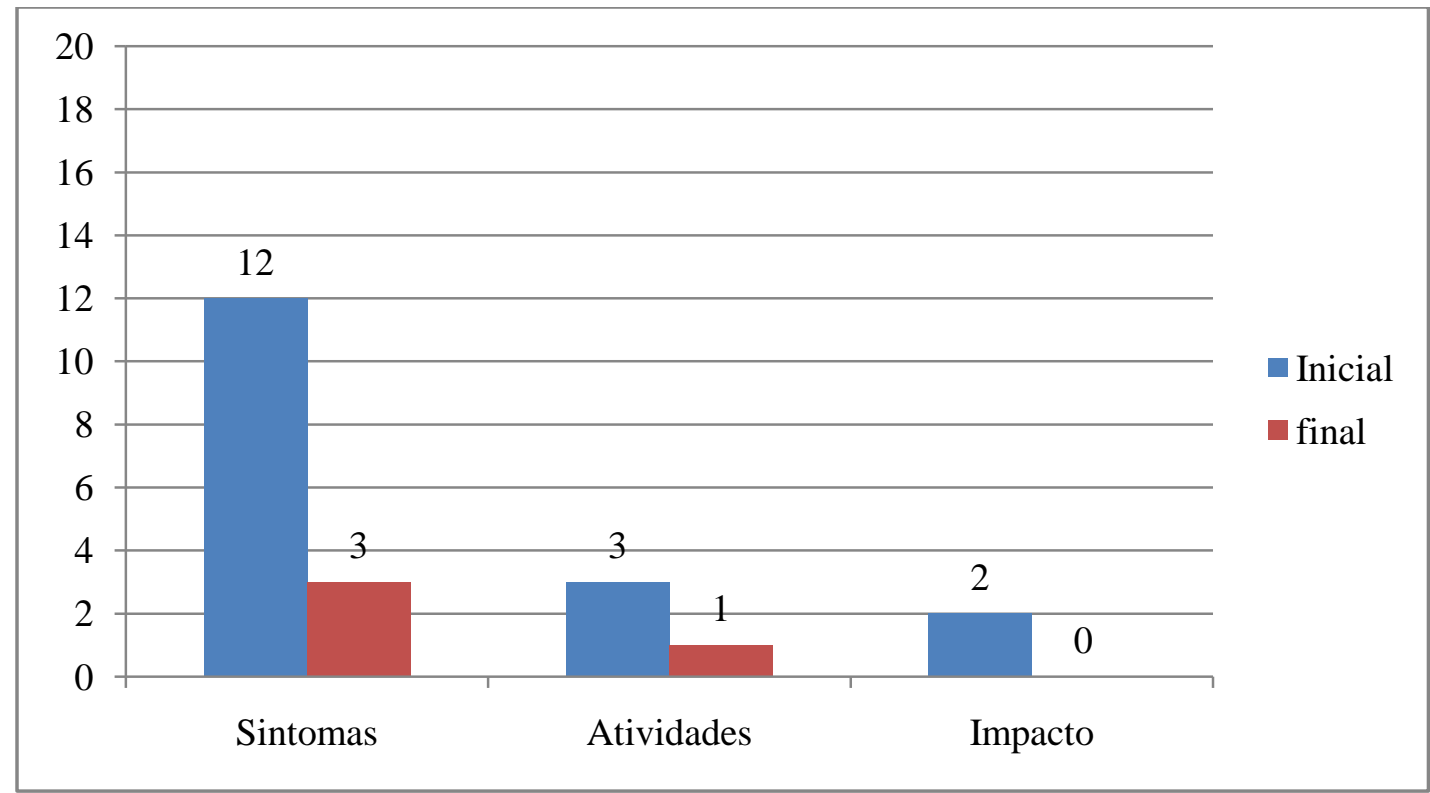

Fonte: elaborada pelos autores

\section{DISCUSSÃO}

Pacientes com DPOC, por se tratar de uma doença de limitação crônica ao fluxo aéreo, tem como característica principal a dispneia, e que em indivíduos debilitados, essa aparece nas atividades do dia-a-dia e até mesmo no repouso e se configura como o principal agente causador do descondicionamento físico (SIMON, 2009). Após a aplicação do protocolo de eletroestimulação respiratória e fisioterapia respiratória e motora convencional, o que sugere uma evolução na força dos músculos intercostais internos, externos e diafragma.

A partir da análise dos resultados observamos que o protocolo de eletroestimulação da musculatura respiratória associada à fisioterapia respiratória e motora convencional apresentou eficácia na reabilitação de paciente com DPOC.

A eletroestimulação é muito útil, principalmente em pacientes que apresentam DPOC grave e disfunção muscular esquelética, os benefícios deste tipo de terapia podem ser particularmente evidentes nos pacientes com dispneia importante, incapazes de se submeterem mesmo a atividades extremamente leves (DOURADO; GODOY, 2004).

A eletroestimulação é normalmente usada na reabilitação de pacientes com patologias neuromusculares, todavia, ultimamente vem sendo utilizada na disfunção muscular esquelética e respiratória. 
Existe um comprometimento irreversível da arquitetura pulmonar, proveniente da DPOC, é a principal razão pela qual os programas de reabilitação pulmonar quando aplicados isoladamente não beneficiarem o quadro de obstrução ao fluxo aéreo. Entretanto, a eletroestimulação auxilia secundariamente na DPOC, diminuindo as disfunções musculares periféricas e respiratórias, anormalidades nutricionais, deficiências cardiovasculares, distúrbios esqueléticos, sensoriais e psicossociais e assim constatando uma melhora no quadro clinico dos pacientes (RODRIGUES, 2010).

Sabe-se que a DPOC está diretamente ligada a redução na $\mathrm{QV}$ dos indivíduos acometidos, por isso nesse estudo avaliamos a eficácia da aplicação do protocolo de eletroestimulação na melhoria da QV dos voluntários. O SGRQ aborda aspectos relacionados a três domínios: sintomas, atividade e impactos psicossociais que a doença respiratória inflige ao paciente (SOUSA; JARDIM; JONES, 2000).

Analisando os gráficos nota-se que a realização do protocolo propiciou uma melhora considerável na QV de ambos os pacientes em todos os campos de avaliação. Diante disto, podemos ponderar que a abordagem terapêutica alternativa, foi viável e de grande relevância no tratamento de pacientes com DPOC, contribuindo de forma satisfatória na melhora da QV dos pacientes.

Um estudo de um programa de reabilitação pulmonar domiciliar previamente, mas que não utilizou-se uso o recurso da eletroestimulação, também obteve melhora em componentes físicos como a capacidade física, dor e estado geral de saúde dos seus pacientes, sendo portanto que não podemos atribuir os nossos achados isoladamente somente a este recuso (SILVA, 2011).

Em uma pesquisa prévia, onde avaliaram-se 15 pacientes com DPOC grave submetidos a eletroestimulação da musculatura respiratória e evidenciaram melhora significativa da função muscular, da tolerância ao exercício e do componente de dispneia do questionário de QV (NEDER, 1999).

O presente trabalho foi realizado a partir da aplicação de um protocolo de eletroestimulação respiratória associado à fisioterapia respiratória e motora convencional, e da utilização do questionário do hospital Saint George de QV em dois pacientes com DPOC. Assim, concluímos que, a realização dos procedimentos supracitados promoveu um aumento das pressões respiratórias máximas, que refletiram diretamente a força muscular respiratória, confirmando que esse recurso pode ser utilizado, com segurança, na prática da 
fisioterapia respiratória, além de possuir efeitos positivos na QV de ambos os pacientes, principalmente nos quesitos atividades e impacto.

Sugerimos a realização de novos estudos, com maior número de participantes,

\section{REFERÊNCIAS}

1. FERNANDES, A. B. S. Reabilitação respiratória em DPOC: a importância da abordagem fisioterapêutica. SOPTER., v. 1, n. 1, p. 71-78, 2009.

2. ARAUJO,J. M.; SANTOS, E. Dois protocolos distintos de reabilitação pulmonar em pacientes portadores de doença pulmonar obstrutiva crônica. Relato de casos e revisão de literatura. RevBrasClinMed, v.10, p. 1, 87-90, 2012.

3.Sociedade Brasileira de Pneumologia e Tisiologia [homepage on the Internet]. Brasília: Sociedade Brasileira de Pneumologia e Tisiologia. DPOC e Saúde Pública - Atendendo as necessidades dos pacientes.(2012).

4. SILVA, E. G.; VIEIRA, D. Estimulação Diafragmática Elétrica Transcutânea na melhora do metabolismo da musculatura respiratória: revisão. Rev MinCi Saúde, v.1, n. 1, p. 69-80, 2009.

5. SOUSA, T. C.; JARDIM J. R.; JONES, P. Validação do questionário do hospital Saint George na doença respiratória em pacientes portadores de DPOC no Brasil. J Pneumol. v. 26, n. 3, p. 119-128, 2000.

6. CANCELLIERO,K. M.; COSTA,D.; SILVA, C. A. Estimulação diafragmática elétrica transcutânea Melhora as condições metabólicas utilizando a eletroestimulação no tratamento de disfunções respiratórias, fazendo com que esse procedimento seja mais utilizado no meio acadêmico e profissional

dos músculos Respiratórios de ratos. Rev. bras. Fisioter, v. 10, n. 1, p. 59-65, 2006.

7. SIMON, K. M. et al. .Índice Prognóstico de Mortalidade BODE e Atividade Física em Doentes Pulmonares Obstrutivos Crônicos. Rev. Bras. Med. Esporte, v.15, n. 1, p.19-22, 2009.

8. DOURADO, V. Z.; GODOY,I.

Recondicionamento muscular na DPOC: principais intervenções e novas tendências. Rev. Bras. Clin. Med. V. 10, n. 4, p. 331-334, 2004.

10. RODRIGUES, M. L., et al.Corrente russa versus corrente FES na reabilitação de músculos esqueléticos desnervados. Anuário da Produção de Iniciação Científica Discente. n. 13, v. 16, p. 45-55, 2010.

11. SOUSA,T.C.; JARDIM,J.R.; JONES P. Validação do questionário do Hospital Saint George na doença respiratória (SGRQ) em pacientes portadoresde doença pulmonar obstrutiva crônica no Brasil. J Pneumol., v. 26, n. 3, p. 119-128, 2000.

12. SILVA, G. P. F. et al. Efeitos de um programa de reabilitação pulmonar domiciliar na qualidade de vida em pneumopatascrônicosestudo piloto. Mov., v. 3, n. 5, p.12-15, 2011.

13. NEDER, J. A. et al. Reference values for lung function tests. I. Static volumes. Braz. J. Med. Biol. Res., v. 32, n. 6, p. 703-71, 1999. 